\title{
Cellulose dissolution and regeneration using a non-aqueous, non-stoichiometric protic ionic liquid system
}

\author{
Laura Berga • Isobel Bruce • Thomas W. J. Nicol (1) - Ashley J. Holding • \\ Noriyuki Isobe $(\mathbb{D} \cdot$ Seishi Shimizu $(\mathbb{C}) \cdot$ Adam J. Walker • Joshua E. S. J. Reid $(1)$
}

Received: 27 January 2020/Accepted: 3 September 2020/Published online: 12 September 2020

(C) The Author(s) 2020

\begin{abstract}
The solubility of cellulose has been studied as a function of composition in the binary mixture of 1,1,3,3-tetramethylguanidine and propionic acid. In amine-rich compositions, greater quantities of cellulose can be dissolved than in the equimolar composition, a.k.a. the protic ionic liquid [TMGH][OPr]. By applying a methodology of a short period of heating followed by cooling, similar concentrations of cellulose can be achieved in a much shorter time period. Finally, regeneration of cellulose from solution can be
\end{abstract}

Electronic supplementary material The online version of this article (https://doi.org/10.1007/s10570-020-03444-8) contains supplementary material, which is available to authorized users.

L. Berga - I. Bruce - T. W. J. Nicol .

S. Shimizu · J. E. S. J. Reid (凹)

York Structural Biology Laboratory, Department of Chemistry, University of York, Heslington,

York YO10 5DD, UK

e-mail: jr649@york.ac.uk

L. Berga · A. J. Holding - A. J. Walker · J. E. S. J. Reid

Worn Again Technologies Ltd, BioCity Nottingham,

Pennyfoot Street, Nottingham NG1 1GF, UK

L. Berga - A. J. Walker · J. E. S. J. Reid

Bioniqs Ltd, BioCity Nottingham, Pennyfoot Street,

Nottingham NG1 1GF, UK

A. J. Holding

Circular Material Solutions Ltd, Lynstock way,

Bolton BL6 4SG, UK achieved by altering the acid:amine molar ratio. In comparison to cellulose regenerated from these solutions using water as an antisolvent, cellulose regenerated with propionic acid exhibit a lower crystallinity as inferred from x-ray diffractometry, but a greater average molecular weight as inferred from gel permeation chromatography.

Keywords Cellulose $\cdot$ Solubility $\cdot$ Protic ionic liquids $\cdot$ Binary mixtures $\cdot$ Solvent effects $\cdot$ XRD

\footnotetext{
N. Isobe

Japan Agency for Marine-Earth Science and Technology (JAMSTEC), Research Institute for Marine Resources Utilization, 2-15 Natsushima-cho, Yokosuka 237-0061, Japan

J. E. S. J. Reid

School of Biosciences - Microbiology, Brewing \& Biotechnology, University of Nottingham, Sutton Bonnington Campus, Loughborough LE12 5RD, Leics, UK
} 


\section{Introduction}

Ionic liquids (ILs) are a class of materials broadly described as salts which have melting points below $100{ }^{\circ} \mathrm{C}$ (Plechkova and Seddon 2008; Hallett and Welton 2011; Austen Angell et al. 2012; Greaves and Drummond 2015). By varying the anion and cation structure, a wide range of properties can be obtained which have led to a number of different applications. Of particular interest is their use as solvents for cellulose processing, as they have been demonstrated to readily dissolve high concentrations of cellulose (Swatloski et al. 2002; Kosan et al. 2008; Wang et al. 2012; Verma et al. 2019). Of the ILs known to dissolve cellulose there are two distinct groups: "aprotic" ILs (AILs) formed of discreet ionic species such as 1-butyl-3-methylimidazolium chloride ([BMIm][Cl]) (Pinkert et al. 2009; Hallett and Welton 2011; Petkovic et al. 2011) and "protic" ILs (PILs) formed from the proton transfer reaction between a Brønsted acid and base such as 1,1,3,3-tetramethylguanidinium propionate ([TMGH][OPr]).(Walker 2004; King et al. 2011; Hauru et al. 2012; Greaves and Drummond 2015; Meenatchi et al. 2017; Becherini et al. 2019). Ionic liquids in mixtures with dipolar aprotic solvents such as dimethyl sulfoxide, $\gamma$-valerolactone and 1-methylimidazole have also been shown to solubilize cellulose quite readily (Xu et al. 2013; Zhao et al. 2013; Gale et al. 2016; Holding et al. 2017), which has greatly enabled the spinning of cellulose fibers using ionic liquids (Hauru et al. 2014; Sixta et al. 2015; Ma et al. 2019). Spinning of fibres requires extrusion of highly viscous cellulose solution into a coagulant bath (Hauru et al. 2014, 2016; Sixta et al. 2015). There the cellulose undergoes regeneration (Medronho and Lindman 2015), which uses antisolvent to induce precipitation (Lindman et al. 2017). Hence, solvents mix with anti-solvent in the process.

The reality of an industrial application of an IL as a solvent for cellulose processing is greatly limited by certain obstacles, typically (1) the number of synthesis steps and ultimately the cost to manufacture the IL (Clark and Tavener 2007; Jessop 2011; George et al. 2015), (2) the potential hazards to both operators and the environment posed by the IL such as toxicity, biodegradability and mutagenicity (Docherty et al. 2006; Jordan and Gathergood 2015; Reid et al. 2015; Clarke et al. 2018) and (3) the purification of the IL from its mixtures with other solvents, such as antisolvents used to regenerate cellulose from its solution (Seddon et al. 2000). In general, PILs address the first of these points as they can be prepared for much lower costs than AILs due to their straightforward preparation (George et al. 2015). Paired with the suggested low microbial toxicity and biodegradability of PILs with the 1,1,3,3-tetramethylguanadinium cation (Pratap Singh et al. 2017) and carboxylic anions (Reid et al. 2018) the use of PIL systems such as $[\mathrm{TMGH}][\mathrm{OPr}]$ for cellulose processing has clear advantages over their AIL counterparts.

The separation of antisolvent from IL remains a common problem in both PIL and AIL based cellulose dissolution processes. For example, small quantities of water significantly reduces the ability for AILs and PILs to dissolve cellulose (Mazza et al. 2009; Hauru et al. 2012). The complete separation of water from ILs is very challenging, due in a large part to water becoming more and more discrete at diminishing concentrations in ILs (Reid et al. 2017b). Additionally, it has recently been shown that many superbases including TMG are susceptible to hydrolysis in aqueous mixtures, raising concern over the applicability of water as an antisolvent for TMG-based cellulose/PIL solutions (Hyde et al. 2019). The use of alternative antisolvents such as compressed $\mathrm{CO}_{2}$ show a potentially facile separation of antisolvent from IL, yet the processing costs of high pressure $\mathrm{CO}_{2}$ in general make their applicability less feasible (Sun et al. 2014). A range of molecular solvents including (but not limited to) acetone, ethanol and dimethyl sulfoxide, have also been employed to regenerate cellulose from ionic liquid solutions (Gupta et al. 2013; Huo et al. 2013; Holding et al. 2014, 2017).

One potential solution to antisolvent separation from a PIL may lie in exploiting the nature of PILs as binary mixtures of a Brønsted acid and base. We have recently shown that changing the stoichiometry in a binary mixture of acetic acid and $N, N$-dimethylethanolamine has a profound effect on the apparent solvent-solute interactions (Reid et al. 2019). Applying that same methodology to the system of propionic acid (HOPr) and 1,1,3,3-tetramethylguanidine (TMG), we show how changing the composition can lead to an increase in cellulose solubility, as well as a means of regenerating cellulose from solution without the need of a dedicated antisolvent. 


\section{Results and discussion}

Viscosity of binary mixtures of 1,1,3,3tetramethylguanidine and propionic acid

Prior to performing the trials of cellulose dissolution, we wanted to characterise the viscosity of the mixtures, as it is an important property for their application as solvents (Marcus 1998; Reid et al. 2017a). Unfortunately, due to the high melting point of the equimolar PIL, we were unable to accurately measure its viscosity within the operating temperatures of our equipment. For all other mixtures and the pure TMG and $\mathrm{PrOH}$ we successfully recorded the viscosity as a function of temperature between 298.15 K and 318.15 K (Fig. 1). Generally speaking, we find that the acid-rich compositions have greater viscosities than the corresponding amine rich mixtures, with the 0.4 Amine mole fraction mixture exhibiting the highest viscosities of all the mixtures studied in this temperature range.

Dissolution of cellulose

The solubility of cellulose in a binary mixture of HOPr and TMG is significantly affected by the solvent mixture composition. We have found that microcrystalline cellulose (MCC) is completely insoluble in all acid rich compositions of these mixtures, as well as the pure precursor materials. Dissolution was confirmed using microscopy to observe any undissolved crystals of the cellulose which could not be observed by the human eye. Surprisingly, the solubility of cellulose

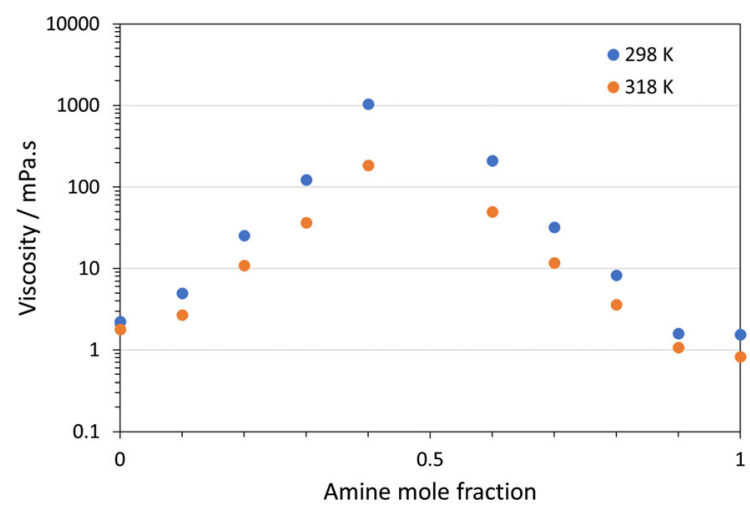

Fig. 1 Viscosity of binary mixtures of HOPr and TMG as a function of amine mole fraction at 298 and $318 \mathrm{~K}$. The full set of experimental data can be found in the supporting information was greater in the 60:40 molar ratio mixture of TMG and $\mathrm{HOPr}(12.5 \mathrm{wt} \%)$ than in the equimolar system (10 wt $\%)$. Furthermore, the $70: 30$ molar ratio mixture could dissolve a large concentration of MCC (7.5 wt $\%$ ) but higher molar ratios of TMG to HOPr could not dissolve MCC.

That an amine-rich composition of TMG-HOPr can dissolve a greater quantity of cellulose than the equimolar composition is likely due to the increased hydrogen bond basicity of the binary mixture. Certainly, it is well understood that a balance of hydrogen bond basicity and hydrogen bond acidity plays an active role in cellulose solubility (Hauru et al. 2012; Parviainen et al. 2013). It is likely that a combination of the propionate anion and neutral TMG result in an increase in cellulose solubility. To explore this hypothesis further, the temperature dependence of cellulose solubility was also studied, as varying the temperature can significantly affect the proton transfer equilibrium in acid-base binary mixtures (Abbott et al. 2018).

We found that cellulose solubility under isothermal conditions is also highly dependent on temperature as well as on composition (Fig. 2). By raising the temperature from $20{ }^{\circ} \mathrm{C}$ to $120{ }^{\circ} \mathrm{C}$, we find the optimum temperature to be $80{ }^{\circ} \mathrm{C}$ for all systems. Above this temperature, cellulose solubility decreases due probably to a shift in the proton transfer equilibrium, resulting in the formation of neutral species with subsequently lower hydrogen bond basicity (Reid et al. 2017a).

Typically, the PIL [TMGH][OPr] has a melting point of around $63{ }^{\circ} \mathrm{C}$ (King et al. 2011), yet the solvent appeared stable even at $40{ }^{\circ} \mathrm{C}$ with gradual cooling, demonstrating supercooling common in PILs (Angell et al. 2007). However the amine-rich mixtures, which were liquid at all temperatures studied, were able to dissolve more cellulose at lower temperatures as well (Fig. 2).

We also explored the solubility of cellulose in the $55 \%$ and $65 \%$ molar percentage of TMG mixtures and find a smooth trend in composition dependence on cellulose solubility, with $60 \%$ molar percentage of TMG having the greatest cellulose solubility (Fig. 2). When held under isothermal conditions at $80{ }^{\circ} \mathrm{C}$ for $15 \mathrm{~h}$, the maximum concentration of MCC we could obtain was $17.5 \mathrm{wt} \%$.

A chance discovery during this work was that after a short period of heating, followed by subsequent 


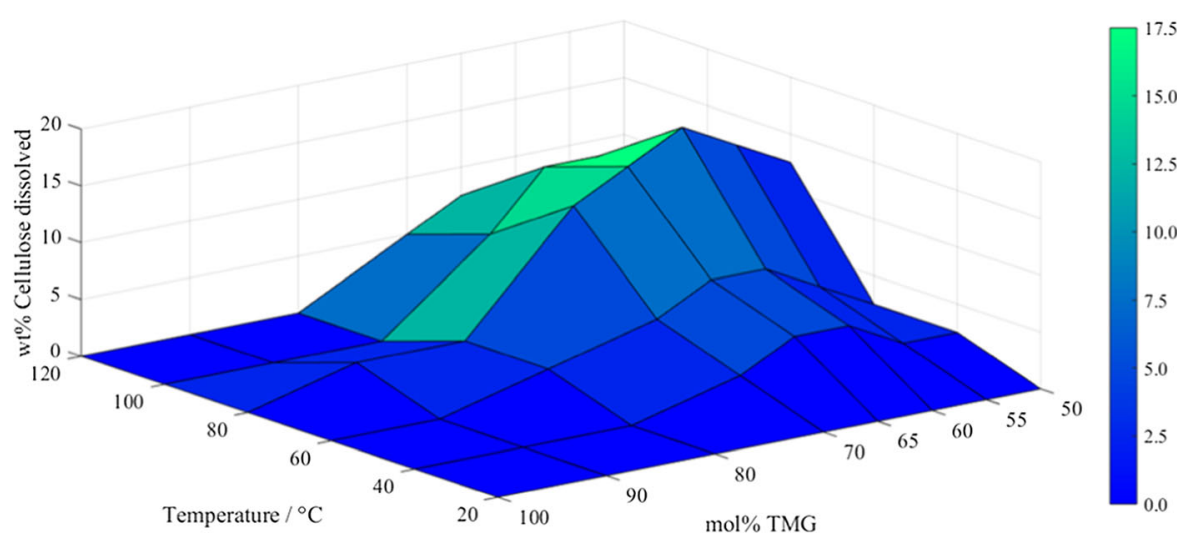

Fig. 2 Dependence of composition and temperature in the TMG-HOPr binary mixture on the solubility of cellulose after $15 \mathrm{~h}$ under isothermal conditions

cooling to room temperature, it was possible to obtain cellulose solutions in a much shorter period of time than previously believed. From this, we pursued a systematic study on heating systems at two temperatures $\left(80{ }^{\circ} \mathrm{C}\right.$ and $\left.100{ }^{\circ} \mathrm{C}\right)$ for a set period of time $(2 \mathrm{~h})$, followed by removing from the vial block to cool to room temperature (Table 1). As a control, samples of each TMG-HOPr mixture screened were heated for $2 \mathrm{~h}$ at $100{ }^{\circ} \mathrm{C}$ and analysed using ${ }^{1} \mathrm{H}$ NMR to confirm that the compositions of each mixture had not changed during the solubility screening tests (See supporting information Figures S1 to S8).

This method of cellulose dissolution resulted in a greater solubility of cellulose in each of the four compositions $(50 \%, 55 \%, 60 \%$ and $65 \% \mathrm{TMG})$ at either temperature compared to isothermal conditions over $15 \mathrm{~h}$ (Table 1). For the 50\%, 55\% and 65\% TMG systems, a greater amount of cellulose could be dissolved when heated at $80{ }^{\circ} \mathrm{C}$ compared to $100{ }^{\circ} \mathrm{C}$, with the $65 \%$ TMG system having the same maximum observed cellulose solubility under both conditions. The maximum concentration of MCC obtained using this method, $20 \mathrm{wt} \%$, was achieved using the 55\% and $60 \%$ TMG mixtures after a total time of $3 \mathrm{~h}$. As expected, these high concentration cellulose solutions had an appearance that was more gel-like as opposed to a free flowing solution; with better mixing technology it may even be possible to obtain greater cellulose solubilities in the 55\% and 60\% TMG mixtures. While there was the development of a yellow colour to some cellulose solutions in both methods of dissolution, there was no discernible trend on the formation of this solution colour with cellulose concentration, acidamine ratio or duration of the heating condition.

Why this cooling step, along with the temperature of heating, is important towards cellulose solubilisation is not straightforward. While the ionic nature certainly plays an important role in the mechanism of cellulose solubilisation, the thermodynamics and kinetics of this heat/cool dissolution process when compared to isothermal conditions requires further experimental and theoretical investigation, which is currently underway in our laboratory.

The increase in solubility with temperature can be explained through the temperature dependence of cellulose disentanglement and decrystallisation (Ghasemi et al. 2017a). Free energy of mixing is touted as an overarching driving force of dissolution (Lindman et al. 2017). The physical processes within the cellulose dissolution mechanism that most closely correspond to this driving force are solvent diffusivity and chain disentanglement (Ghasemi et al. 2017a, b). Ghasemi et al. have shown that solvent diffusivity and chain disentanglement predominantly affect swelling and dissolution, respectively. In order for cellulose molecules to remain in solution after disentanglement, they must be stabilised in solution. In cellobiose and other short chain cellulose molecules, the molecular interactions which provide this stabilisation consist of "charging up" caused by ion accumulation around the molecule in solution (Medronho and Lindman 2015; Bialik et al. 2016; Nicol et al. 2017). These molecular interactions could be present in cellulose. In fact, ion accumulation has been observed in simulation studies of ILs; [Bmim]Cl shows anion accumulation around 
Table 1 Solubility of cellulose (displayed in weight percentage of cellulose in final solution) as a result of heating at $80^{\circ} \mathrm{C}$ (blue) and $100{ }^{\circ} \mathrm{C}$ (orange) for $2 \mathrm{~h}$ followed by subsequent cooling

\begin{tabular}{|c|c|c|c|c|}
\hline $\begin{array}{l}\text { Cooling } \\
\text { time } \\
\text { / minutes }\end{array}$ & $\begin{array}{l}50 \% \text { TMG } \\
\text { / wt } \%\end{array}$ & $\begin{array}{l}55 \% \text { TMG } \\
\text { / wt } \%\end{array}$ & $\begin{array}{l}60 \% \text { TMG } \\
\text { / wt } \%\end{array}$ & $\begin{array}{l}65 \% \mathrm{TMG} \\
\text { / wt } \%\end{array}$ \\
\hline 0 & 7.5 & 7.5 & 7.5 & 7.5 \\
\hline 10 & 10.0 & 12.5 & 12.5 & 10.0 \\
\hline 20 & 15.0 & 15.0 & 17.5 & 15.0 \\
\hline 30 & 15.0 & 15.0 & 17.5 & 15.0 \\
\hline 45 & 15.0 & 15.0 & 17.5 & 15.0 \\
\hline 60 & 17.5 & 20.0 & 20.0 & 17.5 \\
\hline 75 & 17.5 & 20.0 & 20.0 & 17.5 \\
\hline 90 & 17.5 & 20.0 & 20.0 & 17.5 \\
\hline 105 & 17.5 & 20.0 & 20.0 & 17.5 \\
\hline 120 & 17.5 & 20.0 & 20.0 & 17.5 \\
\hline 0 & 7.5 & 7.5 & 7.5 & 7.5 \\
\hline 10 & 7.5 & 10.0 & 10.0 & 7.5 \\
\hline 20 & 7.5 & 12.5 & 12.5 & 10.0 \\
\hline 30 & 10.0 & 12.5 & 12.5 & 12.5 \\
\hline 45 & 12.5 & 12.5 & 12.5 & 12.5 \\
\hline 60 & 12.5 & 15.0 & 15.0 & 15.0 \\
\hline 75 & 15.0 & 15.0 & 15.0 & 15.0 \\
\hline 90 & 15.0 & 15.0 & 15.0 & 15.0 \\
\hline 105 & 15.0 & 15.0 & 15.0 & 15.0 \\
\hline 120 & 15.0 & 17.5 & 17.5 & 17.5 \\
\hline
\end{tabular}

cellulose as a stabilisation interaction within the IL (Gross et al. 2011). By lowering the temperature of the system, the proton exchange equilibrium will be shifted towards ionic species over neutral species, which in turn would promote greater ion accumulation around cellulose and thus stabilise the cellulose in solution (Reid et al. 2017a; Abbott et al. 2018).

\section{Regeneration of cellulose}

The nil solubility of cellulose in the acid-rich compositions of the TMG-HOPr systems inspires the use of propionic acid as an alternative antisolvent (AS) to water which is commonly used for the same purpose. The separation of excess HOPr from an acid-rich composition of TMG-HOPr is expected to be more straightforward than the separation of water from the PIL, due to the non-zero volatility of the binary mixtures in this study. The equimolar protic ionic liquid, [TMGH][OPr] has already been shown to be readily distillable using high temperature and high vacuum.(King et al. 2011) We attempted to distil an acid-rich mixture of TMG and HOPr and found that we were able to distil pure propionic acid from this acid-rich mixture, indicating the practical applicability of this method. However, we soon realised that to properly assess the feasibility of solvent recovery from this mixture will require a more in-depth study of the vapour-liquid equilibria of this binary mixture, which is beyond the scope of this paper. We hope to address this topic in a future publication.

We have demonstrated that propionic acid can indeed act as an effective antisolvent for cellulose. By injecting a $15 \mathrm{wt} \%$ solution of cellulose at $80{ }^{\circ} \mathrm{C}$ into a precipitation bath at the same temperature using a homogenizer, we were successful in regenerating cellulose as a white, granular powder. The material was then subsequently washed further using the same antisolvent, before being dried using acetone to remove residual TMG and HOPr. When comparing cellulose solutions using $50 \mathrm{~mol} \% \mathrm{TMG}$ and $60 \mathrm{~mol} \%$ TMG, the regeneration process was effectively identical. To compare on the quality of regenerated cellulose, water was used as a reference antisolvent.

To verify the effects of our regeneration process on the molecular weight and polydispersity of cellulose, we performed gel permeation chromatography (GPC), $\mathrm{X}$-ray diffractometry (XRD), thermogravimetric analysis (TGA) and infrared spectroscopy (FT-IR).

From GPC measurements, we have found that all regenerated samples have reduced degree of polymerisation, $\mathrm{DP}_{\mathrm{n}}$ and $\mathrm{DP}_{\mathrm{w}}$, than the reference MCC. Furthermore, samples regenerated from 50\% TMG solutions exhibited lower polydispersity, D, and a greater $\mathrm{DP}_{\mathrm{n}}$ and $\mathrm{DP}_{\mathrm{w}}$ than from the 60\% TMG solutions (Table 2). Also, regenerating cellulose using $\mathrm{PrOH}$ results in higher $\mathrm{DP}_{\mathrm{n}}$ and $\mathrm{DP}_{\mathrm{w}}$ than compared to using $\mathrm{H}_{2} \mathrm{O}$, however the polydispersity of regenerated cellulose increases regardless of the regeneration solvent used. (Table 2). From this, we can see that both the stoichiometry of the dissolution media and the choice of antisolvent can have an impact on the properties of the regenerated cellulose polymer chain lengths. Regarding antisolvent, it is well known that 
cellulose can undergo hydrolysis in water, most often in acidic conditions.(Vanhatalo and Dahl 2014) It is therefore likely that by combining water with this cellulose solution during regeneration, hydrolysis of the cellulose takes place. By using $\mathrm{PrOH}$ in place of water as the antisolvent, this mechanism of cellulose molecular weight reduction is avoided, hence the observed trends in $\mathrm{DP}_{\mathrm{n}}$ and $\mathrm{DP}_{\mathrm{w}}$.

These differences due to the choice of antisolvent also affect the thermal degradation behaviour. TGA analysis initially showed that the choice of antisolvent resulted in a slightly different decomposition of cellulose (Fig. 3), which is more apparent when considering the differential of thermogravimetric (DTG) curve (Fig. 4). The DTG curves of each sample showed a maxima rate of loss for volatiles for all regenerated samples at approximately $75^{\circ} \mathrm{C}$. This temperature region is consistent with the boiling point of acetone $\left(56^{\circ} \mathrm{C}\right)$ as oppose to 1,1,3,3-tetramethylguanidine $\left(160-162{ }^{\circ} \mathrm{C}\right)$ or propionic acid $\left(141^{\circ} \mathrm{C}\right)$. The DTG curve showed the maxima rate of mass loss on decomposition to be dependent on the regeneration solvent and not the composition of the dissolution solvent. Furthermore, all regenerated cellulose materials underwent decomposition at lower temperatures than the reference MCC material. This is most likely due to a change in the crystalline allomorph and crystallinity of the cellulose as a result of dissolution and regeneration.(Kilpeläinen et al. 2007; Wang et al. 2013).

The change of cellulose crystalline allomorph as it undergoes dissolution and regeneration is a wellunderstood phenomena (Kilpeläinen et al. 2007; Ciolacu et al. 2011). By performing XRD analysis of the regenerated cellulose materials and the precursor microcrystalline cellulose, we demonstrate that cellulose has undergone a change in crystalline allomorph from cellulose I to II through our process steps (Fig. 5). Specifically, the presence of major peaks at a $2 \theta$ scattering angle of approximately $15^{\circ}, 20^{\circ}$ and $22.5^{\circ}$ in the MCC sample, consistent with the crystal structure of cellulose I (Nishiyama et al. 2002; French 2014; Liu et al. 2015), are absent in all of the regenerated cellulose samples, regardless of the regeneration antisolvent used. The types of antisolvent had a great impact on the crystallinity of the regenerated materials. The cellulose materials regenerated with water showed typical profiles of cellulose II (Langan et al. 2001; Ciolacu et al. 2011; French 2014; Liu et al. 2015), whereas regeneration using propionic acid results in amorphous structure as evidenced by the absence of clear peaks in the scattering profiles. This picture is consistent with the observed differences in the DTG and TGA curves.

Infrared spectroscopy is often employed as a complementary technique for XRD analysis. The change in crystalline allomorph and crystallinity can be captured with the peak shape and position in the FTIR spectra due to the difference in hydrogen bonding network between cellulose I and II, specifically in the $3000-3500 \mathrm{~cm}^{-1}$ region, where the information on the O-H groups are included (Marrinan and Mann 1956; Carrillo et al. 2004; Makarem et al. 2019). The MCC showed typical spectra of cellulose I as evidenced by the two sharp peaks at 3400 and $3350 \mathrm{~cm}^{-1}$ (Marrinan and Mann 1956; Carrillo et al. 2004; Makarem et al. 2019). The samples regenerated by water showed two striking peaks at 3490 and $3450 \mathrm{~cm}^{-1}$ that correspond to the intramolecular hydrogen bonds in cellulose II crystal (Marrinan and Mann 1956; Carrillo et al. 2004; Makarem et al. 2019), indicating that the samples regenerated by water had crystalline cellulose II structure. However, these peaks were not observed in the samples regenerated from propionic acid,

Table 2 Gel Permeation Chromatography (GPC) analysis of the regenerated cellulose materials and the reference starting material, MCC

\begin{tabular}{llrr}
\hline Sample & Polydispersity (D) & DP $_{\mathrm{n}}$ & DP $_{\mathrm{w}}$ \\
\hline Reference MCC & 1.41 & $97 \pm 5$ & $137 \pm 10$ \\
$50 \%$ TMG, PrOH AS & 1.58 & $82 \pm 5$ & $135 \pm 10$ \\
$60 \%$ TMG, PrOH AS & 1.75 & $71 \pm 4$ & $124 \pm 8$ \\
$50 \%$ TMG, $\mathrm{H}_{2} \mathrm{O}$ AS & 1.56 & $77 \pm 4$ & $120 \pm 9$ \\
$60 \%$ TMG, $\mathrm{H}_{2} \mathrm{O}$ AS & 1.68 & $58 \pm 5$ & $94 \pm 8$ \\
\hline
\end{tabular}




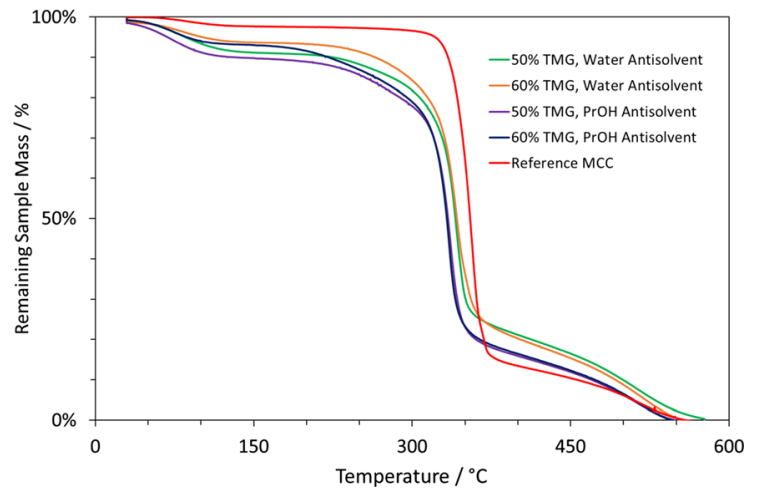

Fig. 3 TGA thermograms of the recovered cellulose materials and reference microcrystalline cellulose. Individual thermograms of each sample can be located in the supporting information

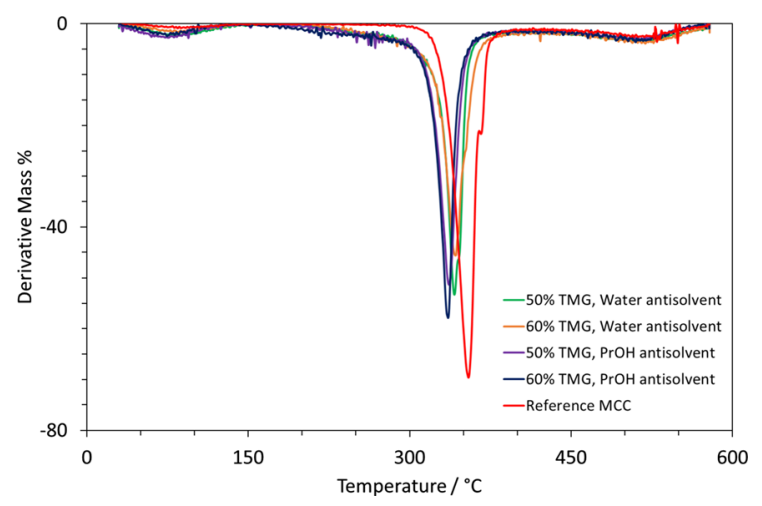

Fig. 4 DTG thermograms of the recovered cellulose materials and reference microcrystalline cellulose. Individual thermograms of each sample can be located in the supporting information

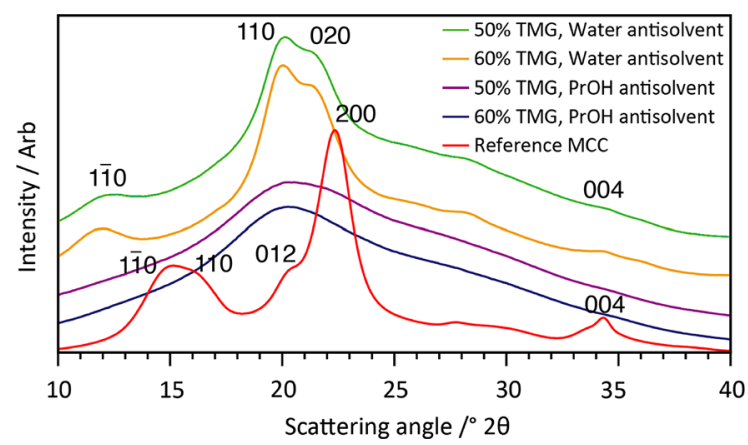

Fig. 5 XRD scattering of the recovered cellulose materials and reference microcrystalline cellulose with assignment of peaks to their corresponding polymorph of cellulose (Langan et al. 2001; Nishiyama et al. 2002) reflecting the amorphous structure. This results further reinforces the structural difference observed in XRD analysis. Additionally, there is a noticeable additional peak around $1600 \mathrm{~cm}^{-1}$ for the regenerated cellulose samples which is not present in the MCC sample. This is most likely due to residual acetone on each regenerated sample as shown by the DTG data (Figs. 4, $6)$.

\section{Conclusions}

To summarise, the dissolution of cellulose has been shown to not be limited to the equimolar composition of 1,1,3,3-tetramethylguanidine and propionic acid; we have found new maximum solubilities of microcrystalline cellulose in a 60:40 molar ratio of the Brønsted base and acid, respectively. By employing a short heating period with a gradual cooling period, up to $20 \mathrm{wt} \%$ MCC solution can be obtained within $3 \mathrm{~h}$ of processing. In addition, exploiting the antisolvent nature of propionic acid, cellulose can be regenerated from these solutions by altering the acid-base ratio towards an acid-rich composition, making additional antisolvent such as water entirely redundant. Thus, our novel solvent media, dissolution conditions and the choice of antisolvent provides a new strategy towards the processing of cellulose in acid-base binary mixtures.

\section{Experimental section}

Propionic acid (HOPr), 1,1,3,3-tetramethylguanidine (TMG) and microcrystalline cellulose (MCC) were purchased from Sigma Aldrich and used without further purification.

Typical cellulose solubility experiments the required amount of TMG was charged into a $20 \mathrm{ml}$ scintillation vial, with propionic acid being slowly titrated into the vial with stirring within a fume cupboard to produce the binary mixture. The mixture is sealed in the vial and placed onto a heating block, ensuring there are no cold spots on the reaction vial. The mixture is then brought to temperature and MCC is added, producing a final mass of solution of $10 \mathrm{~g}$. Dissolution is confirmed by 1) visual inspection of the solution turbidity and 2) microscope image at $40 \times$ magnification of the cellulose solution to 


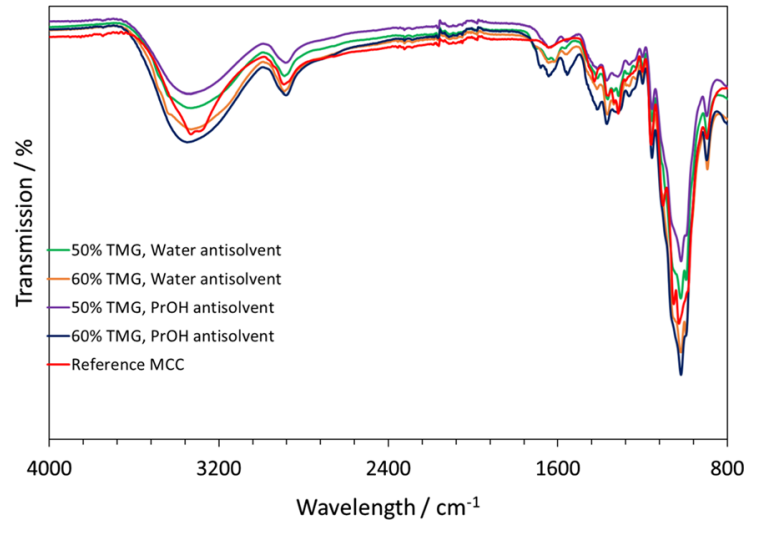

Fig. 6 Infrared spectra of the recovered cellulose materials and reference microcrystalline cellulose. Individual spectra of each sample can be located in the supporting information

confirm whether any undissolved MCC can be observed. Cellulose solubility was tested in intervals of $2.5 \mathrm{wt} \%$ of the total solution.

Typical regeneration experiment cellulose solution (approximately $8 \mathrm{~g}$ ) at $80{ }^{\circ} \mathrm{C}$ was transferred to a $20 \mathrm{ml}$ syringe, where it was then injected into a precipitation solution (approximately $25 \mathrm{~g}$ ) at $80{ }^{\circ} \mathrm{C}$ with constant high shear mixing from a homogenizer. Once all cellulose solution has been injected, the solution was homogenized for $10 \mathrm{~min}$ to ensure complete precipitation has taken place, producing a suspension of cellulose in acid-rich binary mixture/ acid-amine-water tertiary mixture. The solution was then vacuum filtered and washed with a further $2 \times 25 \mathrm{~g}$ of antisolvent before being left to dry under vacuum for $1 \mathrm{~h}$. Samples were then suspended in acetone (approximately $20 \mathrm{~g}$ ) at room temperature before being dried under vacuum for a further $2 \mathrm{~h}$.

Gel permeation chromatography The method outlined is similar to the method used by Silbermann.(Silbermann et al. 2017) The sample, (approximately $0.03 \mathrm{~g}$ ) was swollen overnight in DMSO. The solvent was then exchanged for DMAc by rinsing $\times 3$ with the solvent over vacuum. The solvent-wet sample was placed in $3 \mathrm{ml}$ of $8.7 \mathrm{w} / \mathrm{v} \% \mathrm{DMAc} / \mathrm{LiCl}$ and stirred overnight at room temperature. $0.2 \mathrm{ml}$ of the sample was diluted with $1.9 \mathrm{ml}$ pure DMAc and filtered through a $0.2 \mu \mathrm{m}$ PTFE syringe filter into an autosampler vial, prior to injection. The GPC samples were run on a PSS SECurity system (Agilent 1260 Infinity II) with a SLS 7100 MALLS detector and RI detection for determination of slice concentration, with $2 \times$ PSS
GRAM Columns and 1 Pre-Column. Flow rate was $1.0 \mathrm{ml} / \mathrm{min}$, injection volume was $20 \mu \mathrm{L}$ and the resulting MMD was calculated using the PSS Injected Mass method in WinGPC, using the accurate concentration and on-line determination of $\mathrm{dn} / \mathrm{dc}$, assuming that the entire sample mass had eluted. Results are an average of 3 injections, with the standard deviation of the values used to represent the margin of error in the samples.

Thermogravimetric analysis Samples were analysed using a PerkinElmer TGA4000 instrument. Approximately $10 \mathrm{mg}$ of material was used for each analysis. Each sample was heated at $20{ }^{\circ} \mathrm{C} \mathrm{min}-1$ from $30{ }^{\circ} \mathrm{C}$ to $600{ }^{\circ} \mathrm{C}$ under air. Differential of the thermogravimetric curve was calculated using Pyris software.

$X$-Ray Diffraction Sample measurements were performed using a Samples measurements were carried out by the use of a flat-plate vacuum camera mounted on a rotating anode X-ray generator (Rigaku MircoMax-007HF). The diffraction patterns were recorded at room temperature with Ni-filtered $\mathrm{Cu} \mathrm{K} \alpha$ radiation source $(\lambda=1.5432 \AA, 40 \mathrm{kV}, 30 \mathrm{~mA})$ and Fuji imaging plates (IPs). The sample-to-IP distance was calibrated by the use of $\mathrm{NaF}$ powder $(d=0.23166 \mathrm{~nm})$.

Infrared Spectroscopy Samples were analysed using a PerkinElmer Spectrum 2 FT-IR Spectrometer with an ATR attachment. All samples were measured at $293 \mathrm{~K}$. Spectra recorded are the average of 16 scans in the region $4000-800 \mathrm{~cm}^{-1}$.

${ }^{1} H$ NMR Spectroscopy: Samples were analysed in $\mathrm{D}_{2} \mathrm{O}$ using a Bruker $400 \mathrm{MHz}$ Ultrashield Plus spectrometer with a BBFO probe. All samples were measured at $298.2 \mathrm{~K}$.

Acknowledgments LB gratefully acknowledges funding from the Laidlaw Foundation. TWJN gratefully acknowledges support from an EPSRC studentship grant, the RSC Community Fund and the Gen Foundation. NI acknowledges support from the Daiwa Anglo-Japanese Foundation (Daiwa Foundation Small Grant, ref. 11991/12791) and also from the Grants-inAid for Scientific Research (JSPS KAKENHI Grant Number JP18K18188) from the Japan Society for the Promotion of Science.

Open Access This article is licensed under a Creative Commons Attribution 4.0 International License, which permits use, sharing, adaptation, distribution and reproduction in any medium or format, as long as you give appropriate credit to the original author(s) and the source, provide a link to the Creative Commons licence, and indicate if changes were made. The 
images or other third party material in this article are included in the article's Creative Commons licence, unless indicated otherwise in a credit line to the material. If material is not included in the article's Creative Commons licence and your intended use is not permitted by statutory regulation or exceeds the permitted use, you will need to obtain permission directly from the copyright holder. To view a copy of this licence, visit http://creativecommons.org/licenses/by/4.0/.

Authors Contribution All authors contributed to the study conception and design. Material preparation, data collection and analysis were performed by Laura Berga, Isobel Bruce, Ashely Holding, Noriyuki Isobe and Joshua Reid. The first draft of the manuscript was written by Laura Berga and Joshua Reid, all authors commented on previous versions of the manuscript. All authors read and approved the final manuscript.

\section{References}

Abbott A, Aldous L, Borisenko N et al (2018) Electrochemistry: general discussion. Faraday Discuss 206:405-426. https:// doi.org/10.1039/C7FD90093G

Angell CA, Byrne N, Belieres JP (2007) Parallel developments in aprotic and protic ionic liquids: physical chemistry and applications. ACC Chem Res 40:1228-1236. https://doi. org/10.1021/ar7001842

Austen Angell C, Ansari Y, Zhao Z (2012) Ionic liquids: past, present and future. Faraday Discuss 154:9-27. https://doi. org/10.1039/C1FD00112D

Becherini S, Mezzetta A, Chiappe C, Guazzelli L (2019) Levulinate amidinium protic ionic liquids (PILs) as suitable media for the dissolution and levulination of cellulose. New J Chem 43:4554-4561. https://doi.org/10.1039/ C9NJ00191C

Bialik E, Stenqvist BB, Fang Y et al (2016) Ionization of cellobiose in aqueous alkali and the mechanism of cellulose dissolution. J Phys Chem Lett 7:5044-5048. https://doi. org/10.1021/acs.jpclett.6b02346

Carrillo F, Colom X, Suñol JJ, Saurina J (2004) Structural FTIR analysis and thermal characterisation of lyocell and viscose-type fibres. Eur Polym J 40:2229-2234. https://doi. org/10.1016/j.eurpolymj.2004.05.003

Ciolacu D, Ciolacu F, Popa VI (2011) Amorphous cellulosestructure and characterization. Cellulose Chem Technol 45(1):13-21

Clark JH, Tavener SJ (2007) Alternative solvents: shades of green. Org Process Res Dev 11:149-155. https://doi.org/ 10.1021/op060160g

Clarke CJ, Tu W-C, Levers O et al (2018) Green and sustainable solvents in chemical processes. Chem Rev 118:747-800. https://doi.org/10.1021/acs.chemrev.7b00571

Docherty KM, Hebbeler SZ, Kulpa CF Jr, CF, (2006) An assessment of ionic liquid mutagenicity using the Ames Test. Green Chem 8:560-567. https://doi.org/10.1039/ B602418A
French AD (2014) Idealized powder diffraction patterns for cellulose polymorphs. Cellulose 21:885-896. https://doi. org/10.1007/s10570-013-0030-4

Gale E, Wirawan RH, Silveira RL et al (2016) Directed discovery of greener cosolvents: new cosolvents for use in ionic liquid based organic electrolyte solutions for cellulose dissolution. ACS Sustain Chem Eng 4:6200-6207. https://doi.org/10.1021/acssuschemeng.6b02020

George A, Brandt A, Tran K et al (2015) Design of low-cost ionic liquids for lignocellulosic biomass pretreatment. Green Chem 17:1728-1734. https://doi.org/10.1039/ C4GC01208A

Ghasemi M, Alexandridis P, Tsianou M (2017a) Cellulose dissolution: insights on the contributions of solvent-induced decrystallization and chain disentanglement. Cellulose 24:571-590. https://doi.org/10.1007/s10570-0161145-1

Ghasemi M, Singapati AY, Tsianou M, Alexandridis P (2017b) Dissolution of semicrystalline polymer fibers: numerical modeling and parametric analysis. AIChE J 63:1368-1383. https://doi.org/10.1002/aic.15615

Greaves TL, Drummond CJ (2015) Protic ionic liquids: evolving structure-property relationships and expanding applications. Chem Rev 115:11379-11448. https://doi.org/10. 1021/acs.chemrev.5b00158

Gross AS, Bell AT, Chu J-W (2011) Thermodynamics of cellulose solvation in water and the ionic liquid 1-butyl-3methylimidazolim chloride. J Phys Chem B 115:13433-13440. https://doi.org/10.1021/jp202415v

Gupta KM, Hu Z, Jiang J (2013) Cellulose regeneration from a cellulose/ionic liquid mixture: the role of anti-solvents. RSC Adv 3:12794-12801. https://doi.org/10.1039/ c3ra40807h

Hallett JP, Welton T (2011) Room-temperature ionic liquids. Solvents for synthesis and catalysis. 2. Chem Rev 111:3508-3576. https://doi.org/10.1021/cr1003248

Hauru LKJ, Hummel M, King AWT et al (2012) Role of solvent parameters in the regeneration of cellulose from ionic liquid solutions. Biomacromol 13:2896-2905. https://doi. org $/ 10.1021 / \mathrm{bm} 300912 \mathrm{y}$

Hauru LKJ, Hummel M, Michud A, Sixta H (2014) Dry jet-wet spinning of strong cellulose filaments from ionic liquid solution. Cellulose 21:4471-4481. https://doi.org/10.1007/ s10570-014-0414-0

Hauru LKJ, Hummel M, Nieminen K et al (2016) Cellulose regeneration and spinnability from ionic liquids. Soft Matter 12:1487-1495. https://doi.org/10.1039/ c5sm02618k

Holding AJ, Heikkilä M, Kilpeläinen I, King AWT (2014) Amphiphilic and phase-separable ionic liquids for biomass processing. Chemsuschem 7:1422-1434. https://doi.org/ 10.1002/cssc.201301261

Holding AJ, Parviainen A, Kilpeläinen I et al (2017) Efficiency of hydrophobic phosphonium ionic liquids and DMSO as recyclable cellulose dissolution and regeneration media. RSC Adv 7:17451-17461. https://doi.org/10.1039/ c7ra01662j

Huo F, Liu Z, Wang W (2013) Cosolvent or antisolvent? A molecular view of the interface between ionic liquids and cellulose upon addition of another molecular solvent. 
J Phys Chem B 117:11780-11792. https://doi.org/10.1021/ jp407480b

Hyde AM, Calabria R, Arvary R et al (2019) Investigating the underappreciated hydrolytic instability of 1,8-diazabicyclo[5.4.0] undec-7-ene and related unsaturated nitrogenous bases. Org Process Res Dev 23:1860-1871. https://doi.org/ 10.1021/acs.oprd.9b00187

Jessop PG (2011) Searching for green solvents. Green Chem 13:1391-1398

Jordan A, Gathergood N (2015) Biodegradation of ionic liquids-a critical review. Chem Soc Rev 44:8200-8237. https://doi.org/10.1039/c5cs00444f

Kilpeläinen I, Xie H, King A et al (2007) Dissolution of wood in ionic liquids. J Agric Food Chem 55:9142-9148. https:// doi.org/10.1021/jf071692e

King AWT, Asikkala J, Mutikainen I et al (2011) Distillable acid-base conjugate ionic liquids for cellulose dissolution and processing. Angew Chemie Int Ed 50:6301-6305. https://doi.org/10.1002/anie.201100274

Kosan B, Michels C, Meister F (2008) Dissolution and forming of cellulose with ionic liquids. Cellulose 15:59-66. https:// doi.org/10.1007/s10570-007-9160-x

Langan P, Nishiyama Y, Chanzy H (2001) X-ray structure of mercerized cellulose II at $1 \AA$ resolution. Biomacromol 2:410-416. https://doi.org/10.1021/bm005612q

Lindman B, Medronho B, Alves L et al (2017) The relevance of structural features of cellulose and its interactions to dissolution, regeneration, gelation and plasticization phenomena. Phys Chem Chem Phys 19:23704-23718. https:// doi.org/10.1039/C7CP02409F

Liu Z, Sun X, Hao M et al (2015) Preparation and characterization of regenerated cellulose from ionic liquid using different methods. Carbohydr Polym 117:54-62. https:// doi.org/10.1016/j.carbpol.2014.09.053

Ma Y, Zeng B, Wang X, Byrne N (2019) Circular textiles: closed loop fiber to fiber wet spun process for recycling cotton from denim. ACS Sustain Chem Eng. https://doi. org/10.1021/acssuschemeng.8b06166

Makarem M, Lee CM, Kafle K et al (2019) Probing cellulose structures with vibrational spectroscopy. Cellulose 26:35-79. https://doi.org/10.1007/s10570-018-2199-z

Marcus Y (1998) The properties of solvents, 1st edn. Wiley, Chichester

Marrinan HJ, Mann J (1956) Infrared spectra of the crystalline modifications of cellulose. J Polym Sci XXI:301-311

Mazza M, Catana DA, Vaca-Garcia C, Cecutti C (2009) Influence of water on the dissolution of cellulose in selected ionic liquids. Cellulose 16:207-215. https://doi.org/10. 1007/s10570-008-9257-X

Medronho B, Lindman B (2015) Brief overview on cellulose dissolution/regeneration interactions and mechanisms. Adv Colloid Interface Sci 222:502-508. https://doi.org/10. 1016/j.cis.2014.05.004

Meenatchi B, Renuga V, Manikandan A (2017) Cellulose dissolution and regeneration using various imidazolium based protic ionic liquids. J Mol Liq 238:582-588. https://doi. org/10.1016/j.molliq.2016.05.008

Nicol TWJ, Isobe N, Clark JH, Shimizu S (2017) Statistical thermodynamics unveils the dissolution mechanism of cellobiose. Phys Chem Chem Phys 19:23106-23112. https://doi.org/10.1039/C7CP04647B
Nishiyama Y, Langan P, Chanzy H (2002) Crystal structure and hydrogen-bonding system in cellulose $\mathrm{I} \beta$ from synchrotron $\mathrm{X}$-ray and neutron fiber diffraction. J Am Chem Soc 124:9074-9082. https://doi.org/10.1021/ja0257319

Parviainen A, King AWT, Mutikainen I et al (2013) Predicting cellulose solvating capabilities of acid-base conjugate ionic liquids. Chemsuschem 6:2161-2169. https://doi.org/ 10.1002/cssc. 201300143

Petkovic M, Seddon KR, Rebelo LPN, Pereira CS (2011) Ionic liquids: a pathway to environmental acceptability. Chem Soc Rev 40:1383-1403. https://doi.org/10.1039/c004968a

Pinkert A, Marsh KN, Pang S, Staiger MP (2009) Ionic liquids and their interaction with cellulose. Chem Rev 109:6712-6728. https://doi.org/10.1021/cr9001947

Plechkova NV, Seddon KR (2008) Applications of ionic liquids in the chemical industry. Chem Soc Rev 37:123-150. https://doi.org/10.1039/b006677j

Pratap Singh A, Sithambaram D, Sanghavi R et al (2017) Environmentally benign tetramethylguanidinium cation based ionic liquids. New J Chem 41:12268-12277. https:// doi.org/10.1039/C7NJ03167J

Reid JESJ, Bernardes CES, Agapito F et al (2017a) Structureproperty relationships in protic ionic liquids: a study of solvent-solvent and solvent-solute interactions. Phys Chem Chem Phys 19:28133-28138. https://doi.org/10.1039/ c7cp05076c

Reid JESJ, Gammons RJ, Slattery JM et al (2017b) Interactions in water-ionic liquid mixtures: comparing protic and aprotic systems. J Phys Chem B 121:599-609. https://doi. org/10.1021/acs.jpcb.6b10562

Reid JESJ, Prydderch H, Spulak M et al (2018) Green profiling of aprotic versus protic ionic liquids: synthesis and microbial toxicity of analogous structures. Sustain Chem Pharm 7: https://doi.org/10.1016/j.scp.2017.11.001

Reid JESJ, Shimizu S, Walker AJ (2019) Connecting precursors to a protic ionic liquid: Effects of hydrogen bond synergy in acid-base binary mixtures on the solvent-solute interactions. J Mol Liq 111746: https://doi.org/10.1016/j.molliq. 2019.111746

Reid JESJ, Sullivan N, Swift L et al (2015) Assessing the mutagenicity of protic ionic liquids using the mini Ames test. Sustain Chem Process 3:17. https://doi.org/10.1186/ s40508-015-0044-3

Swatloski RP, Spear SK, Holbrey JD, A, Rogers RD, (2002) Dissolution of cellose with ionic liquids. J Am Chem Soc 124:4974-4975. https://doi.org/10.1021/JA025790M

Seddon KR, Stark A, Torres M-J (2000) Influence of chloride, water, and organic solvents on the physical properties of ionic liquids. Pure Appl Chem 72:2275-2287. https://doi. org/10.1351/pac200072122275

Silbermann S, Weilach C, Kliba G et al (2017) Improving molar mass analysis of cellulose samples with limited solubility. Carbohydr Polym 178:302-310. https://doi.org/10.1016/J. CARBPOL.2017.09.031

Sixta H, Michud A, Hauru L et al (2015) Ioncell-F: a highstrength regenerated cellulose fibre. Nord Pulp Pap Res J 30:43-57. https://doi.org/10.3183/npprj-2015-30-01p043-057

Sun X, Chi Y, Mu T (2014) Studies on staged precipitation of cellulose from an ionic liquid by compressed carbon 
dioxide. Green Chem 16:2736-2744. https://doi.org/10. 1039/C4GC00085D

Vanhatalo KM, Dahl OP (2014) Effect of mild acid hydrolysis parameters on properties of microcrystalline cellulose. BioResources 9:4729-4740. https://doi.org/10.15376/ biores.9.3.4729-4740

Verma C, Mishra A, Chauhan S et al (2019) Dissolution of cellulose in ionic liquids and their mixed cosolvents: a review. Sustain Chem Pharm 13:100162. https://doi.org/ 10.1016/j.scp.2019.100162

Walker AJ (2004) Ionic liquids comprising nitrogen containing cations. Eur Pat. 1805131

Wang H, Gurau G, Rogers RD (2012) Ionic liquid processing of cellulose. Chem Soc Rev 41:1519-1537. https://doi.org/ $10.1039 / \mathrm{c} 2 \mathrm{cs} 15311 \mathrm{~d}$

Wang Z, McDonald AG, Westerhof RJM et al (2013) Effect of cellulose crystallinity on the formation of a liquid intermediate and on product distribution during pyrolysis. J Anal Appl Pyrolysis 100:56-66. https://doi.org/10.1016/ j.jaap.2012.11.017

Xu A, Zhang Y, Zhao Y, Wang J (2013) Cellulose dissolution at ambient temperature: role of preferential solvation of cations of ionic liquids by a cosolvent. Carbohydr Polym 92:540-544. https://doi.org/10.1016/j.carbpol.2012.09. 028

Zhao Y, Liu X, Wang J, Zhang S (2013) Insight into the cosolvent effect of cellulose dissolution in Imidazoliumbased ionic liquid systems. J Phys Chem B 117:9042-9049. https://doi.org/10.1021/jp4038039

Publisher's Note Springer Nature remains neutral with regard to jurisdictional claims in published maps and institutional affiliations. 